\title{
CONGRESO DEL ALUMNADO COMO HERRAMIENTA PARA EL DESARROLLO DE HABILIDADES COMPETENCIALES EN LOS GRADOS DE EdUCACIÓN INFANTIL Y PRIMARIA
}

\author{
Adoración Antolí Cabrera* \\ Carolina Pérez Dueñas* \\ Juan Antonio Moriana Elvira \\ $\mathbf{M}^{\mathrm{a}}$ Carmen Tabernero Urbieta \\ $\mathbf{M}^{\mathrm{a}}$ Araceli Sánchez Raya \\ Eliana $\mathbf{M}^{a}$ Moreno Osella \\ $M^{a}$ Salud Jiménez Romero \\ $M^{a}$ del Rosario Castillo Mayén \\ Tamara Gutiérrez Domingo \\ María del Valle Arriaga Díaz
}

aantoli@uco.es and cpduenas@uco.es (*Corresponding Author)

Universidad de Córdoba

\begin{abstract}
According to the literature, one of the main aims of the European Higher Education Area is that students must develop basic competences, being active agents of their own knowledge, emphasizing discovery learning and the role of the professor as a guide. Following this line, we have developed a project of educational innovation which main aim was fostering autonomy and active learning in students. With this purpose, the students followed a learning methodology based on research and promoting a critical attitude rooted in scientific approach.

Thus, the «I Congress of Psychology and Education: the Congress of Students» emerged at the Faculty of Education of the University of Cordoba. A Scientific Congress where the students, who were studying different Psychology subjects in the Bachelor of Early Childhood Education or Bachelor of Primary Education, were the agents of the oral presentations, as well as the scientific posters. The main topic was Educational psychology. In the same line, one year later, it was celebrated the second edition of the Congress. In this edition, we expanded the participation and topics and the methodology was improved.
\end{abstract}

Keywords: Autonomy Learning, Active Learning, Basic competencies, Educational innovation, Critical attitude.

\section{Resumen}

De acuerdo con la literatura, uno de los objetivos fundamentales del Espacio Europeo de Educación Superior es que el alumnado debe desarrollar una serie de competencias, siendo agentes activos de su propio conocimiento, primando el aprendizaje por descubrimiento guiado por el profesor. Siguiendo esta línea, desarrollamos un proyecto de innovación docente con el objetivo de fomentar en los estudiantes una actitud activa y autónoma hacia la adquisición de su propio conocimiento. Para ello, los estudiantes siguieron una metodología de aprendizaje basado en la investigación, potenciando una actitud crítica sustentada en criterios científicos.

Así surgió el «I Congreso de Psicología y Educación: el Congreso de los Estudiantes » llevado a cabo en la Facultad de Ciencias de la Educación de la Universidad de Córdoba. Un Congreso Científico donde los alumnos que cursaban distintas asignaturas de Psicología en los grados de Educación Infantil y Primaria fueron los protagonistas de las presentaciones orales, así como de los pósteres científicos, sobre temáticas cuyo eje fundamental fue la Psicología en el ámbito educativo. Un año más tarde se celebró su segunda edición continuando en la misma línea de actuación. En ésta se consiguió perseverar en los objetivos y perfeccionar la forma de llevar a cabo la experiencia adaptándola a nuevas temáticas y ampliando la participación.

Palabras clave: Aprendizaje autónomo, Aprendizaje activo, Competencias básicas, Innovación docente, Actitud crítica. (máx. 5)

\section{INTRODUCCIÓN}

El objetivo de este proyecto es fomentar en los alumnos una actitud activa y autónoma hacia la adquisición de su propio conocimiento, desarrollando un aprendizaje basado en la investigación y fomentando una actitud crítica sustentada en criterios científicos.

Siguiendo los objetivos fundamentales de la Educación Europea Superior, pretendemos que el alumnado sea el agente activo de su propio conocimiento, primando el aprendizaje por descubrimiento guiado por el profesor (Brauer, 2013; Boyer Comission, 1998). Para lograrlo, nos apoyamos en el desarrollo de una actividad que supone la culminación del proceso de aprendizaje: un congreso científico donde los alumnos que cursan distintas asignaturas de Psicología en los grados de Educación Infantil y Primaria son los protagonistas. Los alumnos desempeñan diferentes roles en el desarrollo del congreso. Son autores de las presentaciones orales, así como de los pósteres científicos y también participan en la organización del propio congreso. Las presentaciones, ya sean en formato oral o póster, versan sobre temáticas cuyo eje fundamental es la Psicología en el ámbito educativo.

La idea es que se trate de un congreso de alumnos para alumnos, teniendo el protagonismo, no sólo del desarrollo de las presentaciones, sino de la propia organización. En este marco, hemos organizado dos ediciones del 
«Congreso de Psicología y Educación: el Congreso de los Estudiantes». La actividad está especialmente dirigida al profesorado y alumnado que imparte y cursa asignaturas de los departamentos de Psicología y Educación. Logramos así abordar su formación y la investigación de los diferentes temas que integran su perfil profesional, desde una perspectiva interdisciplinar. A estos se suman el profesorado y alumnado, tanto de la Facultad de Ciencias de la Educación, como de otras Facultades. De especial relevancia ha sido la participación y colaboración de gran parte de Personal de Administración y Servicios, y también de profesionales externos a la propia Universidad.

En las dos ediciones que hemos celabrado del congreso hemos complementado la propuesta que se hace con la participación de profesionales en ejercicio elegidos por el propio alumnado. De esta forma, además de promover la comunicación y el intercambio de ideas entre profesionales en formación y en activo, motivamos la participación implicándolos en la toma de decisiones sobre la organización. El objetivo es difuminar los límites entre la formación y el ejercicio de la profesión, de forma que el alumnado asuma el rol que posteriormente va a ejercer.

Aunque el congreso sea el foro final para transmitir e integrar conocimientos, durante todo el curso trabajamos para facilitar que el alumnado desarrolle su capacidad de aprender a aprender (De Miguel, 2009), de desarrollar habilidades de comunicación para la transmisión de dicho conocimiento, tanto en expresión oral como escrita, haciendo especial hincapié en el uso creativo de nuevas tecnologías, además de fomentar el aprendizaje de las competencias básicas de los grados de Educación Infantil y Primaria. El trabajo de tutorización, orientación y varios talleres y seminarios, que son impartidos por profesionales en estos ámbitos,ayudarán a la consecución de los objetivos propuestos.

\section{OBjETIVOS}

Anteriormente hemos hecho referencia a los objetivos generales del proyecto, a continuación, pasamos a detallar objetivos específicos del mismo:

1. Potenciar y valorar la autonomía y el rol activo en el propio aprendizaje del alumno.

2. Profundizar en los conocimientos adquiridos en distintas asignaturas de Psicología y Educación recibidas durante los grados de Educación Infantil y Primaria.

3. Eliminar las barreras para una adecuada formación interdisciplinar, uniendo distintas asignaturas en una misma temática de conocimiento.

4. Difundir y compartir los conocimientos sobre Psicología y Educación con la comunidad educativa y profesional utilizando formatos novedosos.

5. Fomentar una actitud crítica sustentada en criterios científicos ante la información que se ofrece y se recibe.

6. Fomentar el plurilingüismo, ya que las presentaciones orales y los pósteres científicos deberán tener un resumen en español y abstract en inglés y su desarrollo podrá ser en ambas lenguas.

7. Aprender a usar de forma creativa las tecnologías de la información mediante las presentaciones, ya sea en formato de comunicación oral o como póster científico.

8. Desarrollar mediante la práctica habilidades de comunicación tanto oral como escrita en el ámbito profesional y científico, ya que uno de los objetivos del sistema educativo actual es conseguir que el alumnado desarrolle competencias y habilidades por sí mismos, que les preparen para enfrentarse con posterioridad al ámbito profesional (Rué, 2009).

9. Desarrollar las cinco competencias básicas contenidas en los planes de estudio de ambas titulaciones:

(CB1): Que los estudiantes demuestren poseer y comprender conocimientos en un área de estudio que parte de la base de la educación secundaria general, y se suele encontrar a un nivel que, si bien se apoya en libros de texto avanzados, incluye también algunos aspectos que implican conocimientos procedentes de la vanguardia de su campo de estudio

(CB2): Que los estudiantes sepan aplicar sus conocimientos a su trabajo o vocación de una forma profesional y posean las competencias que suelen demostrarse por medio de la elaboración y defensa de argumentos y la resolución de problemas dentro de su área de estudio.

(CB3): Que los estudiantes tengan la capacidad de reunir e interpretar datos relevantes (normalmente dentro de su área de estudio) para emitir juicios que incluyan una reflexión sobre temas relevantes de índole social, científica o ética.

(CB4): Que los estudiantes puedan transmitir información, ideas, problemas y soluciones a un público tanto especializado como no especializado.

(CB5): Que los estudiantes desarrollen aquellas habilidades de aprendizaje necesarias para emprender estudios posteriores con un alto grado de autonomía.

10. Cultivar el sentido de comunidad educativa, animando a la participación a todo el alumnado de la Facultad de Ciencias de la Educación, profesores, personal de administración y servicio; y especialmente a colectivos de profesionales relacionados: tutores externos de prácticas, asociaciones colaboradoras, etc. 
11. Intercambiar conocimientos y procedimientos entre estudiantes y profesionales en activo, especialistas en las temáticas abordadas en el congreso, además de facilitar el paso del alumnado a su rol como futuros profesionales.

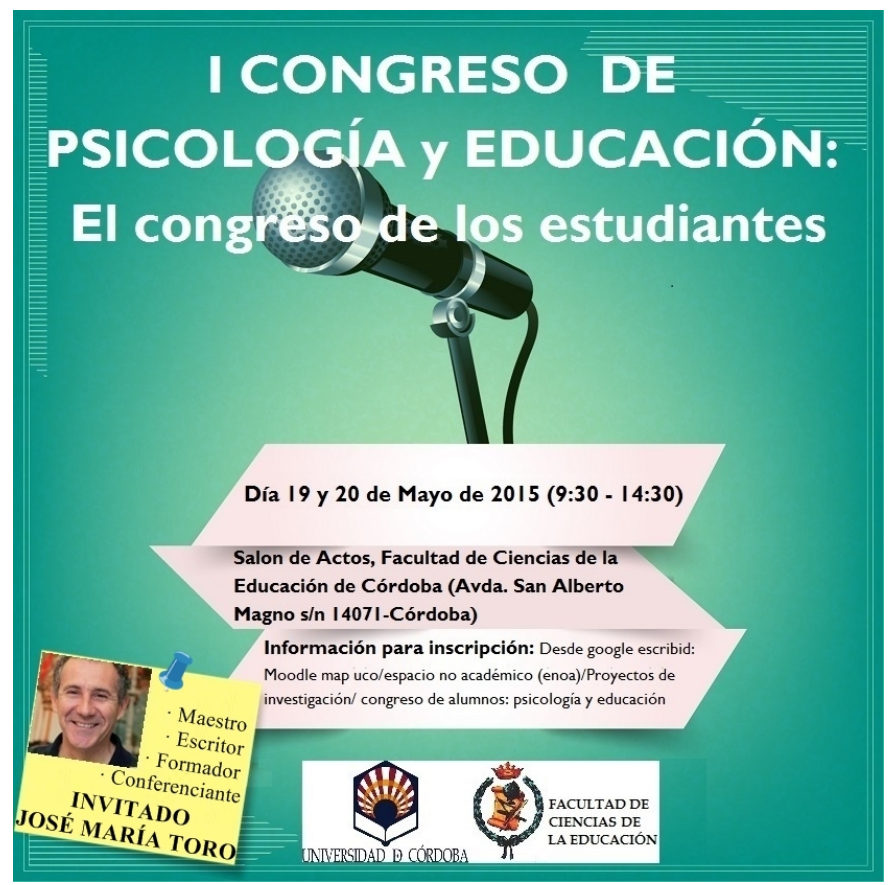

1. Cartel anunciador I Congreso de Psicología y

Educación.

\section{MEtodología}

La metodología empleada en este proyecto pretende conseguir en su mayor parte la adquisición de habilidades:

- Aprendizaje Activo y Autónomo. El aprendizaje centrado en la actividad del alumnado como protagonista en el proceso de aprendizaje (Fink, 2003). El propio estudiante es el que elije el tema sobre el que va profundizar e investigar, para posteriormente exponer.

- Tutorización y Orientación Efectiva, utilizando el feedback constructivo para mejorar la ejecución a través del esfuerzo y la retroalimentación. El profesorado implicado en el proyecto tutoriza y orienta al alumnado en el horario destinado a tal fin.

- Práctica y entrenamiento en Habilidades (Alverno College, 2003). El alumnado "se hace consciente" (metacognición) de qué habilidades tienen más desarrolladas y cuáles deben practicar en mayor medida. Se imparten varios talleres en el horario acordado por el profesorado implicado. En las guías docentes de las asignaturas de Psicología y Educación de los grados de Educación Infantil y Primaria se contemplan la realización de trabajos sobre distintas temáticas. Así, el profesorado participante en el proyecto fomenta la implicaciónde su alumnado en el Congreso, así como la práctica sobre habilidades específicas que se pretenden desarrollar.

- Utilización de la plataforma de enseñanza virtual Moodle como medio de comunicación y base del Congreso. En ella se incluye información relevante, sirviendo de foro para resolver dudas, enviar las propuestas de presentación, difusión de la información relativa al Congreso, etc.

\subsection{ACTIVIDADES DESARROLLADAS}

La principal actividad por parte del alumnado consiste en el desarrollo y la exposición de un trabajo de investigación. Esta actividad principal se desglosa en actividades más concretas: 
1. Selección de un tema específico y concreto, que sea fácil de ilustrar con ejemplos, imágenes y casos prácticos. La tutorización en esta fase es crucial para la orientación del trabajo hacia un tema relevante y abordable por el alumnado.

2. Hacer una búsqueda bibliográfica sobre el tema de interés, utilizando diferentes fuentes de información y eligiendo las referencias de acuerdo a criterios científicos y de calidad.

3. Elaboración y preparación de la presentación oral o póster científico, incluyendo la presentación en grupos pequeños del trabajo realizado. Este trabajo se desarrollará como parte del trabajo en las clases prácticas y tutorías de las distintas asignaturas.

4. Incorporación de sugerencias o cambios al trabajo realizado por el resto de compañeros o tutor, hasta conseguir que tanto el contenido, como la forma en la que se expone, así como los materiales y medios técnicos empleados sean los adecuados a los objetivos.

5. Realización de talleres específicos que tengan como objetivo profundizar en habilidades de comunicación, uso de nuevas tecnologías y búsqueda de información impartido por personal cualificado.

6. Exposición del trabajo en el congreso, cuyo formato será el de los congresos profesionales. El alumnado envía su propuesta de participación a dicho congreso; elabora y redacta un resumen del trabajo que quiere presentar y aporta la bibliografía utilizada. Se pueden presentar tanto comunicaciones orales cortas como pósteres científicos. El comité científico del congreso seleccionade todas las presentadas, aquellas que por su relevancia, originalidad o aplicando criterios de calidad, sean las más adecuadas para su presentación en formato de comunicación oral o póster.

7. Los participantes deben ajustarse al tiempo y forma estipulados en su presentación, donde tienen la oportunidad de mostrar las habilidades adquiridas en las fases previas del proyecto. Los pósteres se presentan en sesiones grupales y permitenal autor exponer verbalmente el contenido a los asistentes.

8. Se fomenta el intercambio de preguntas y de reflexiones tanto en las comunicaciones orales como en las de formato poster.

9. El congreso permite la selección de las mejores presentaciones y pósteres presentados, en modalidad de presentación español e inglés, mediante la votación del público asistente.

10. Al final se les proporciona un diploma de asistencia y depresentacióna los autores, además de un reconocimiento a los ganadores de los premios.

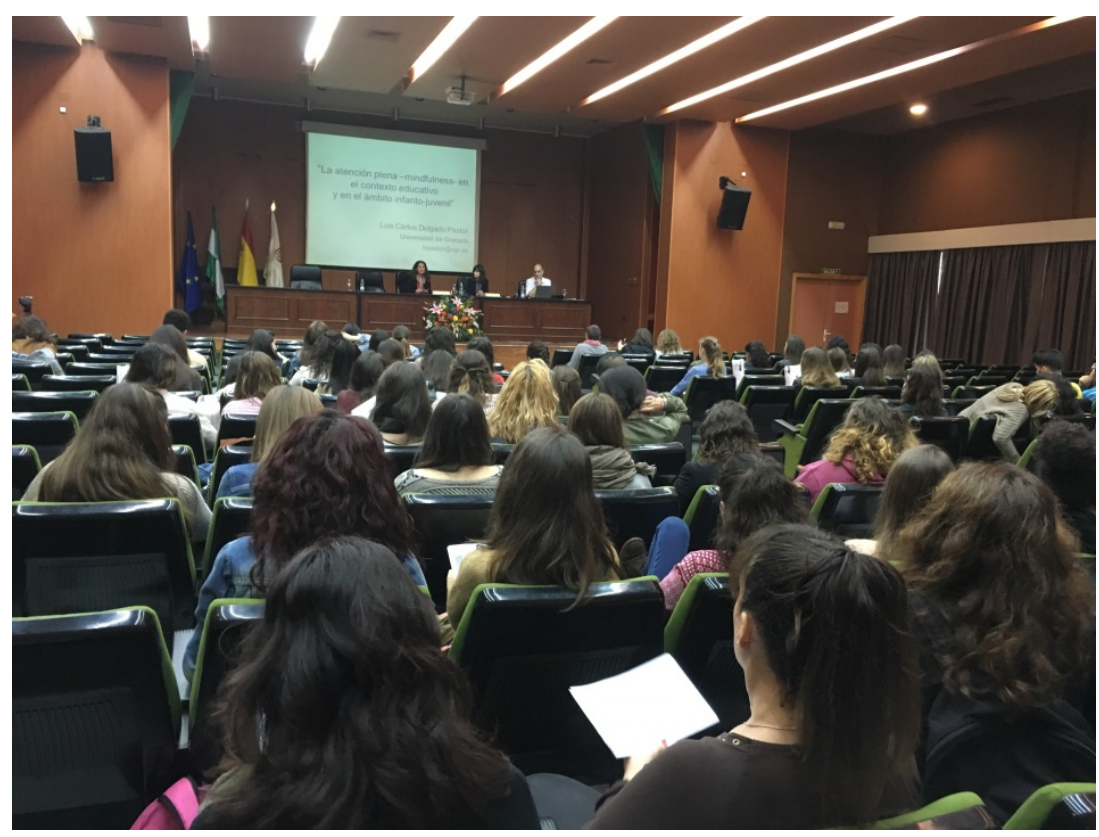

2. Imagen de la inauguración del "II Congreso de Psicología y Educación: El Congreso de los Estudiantes". 


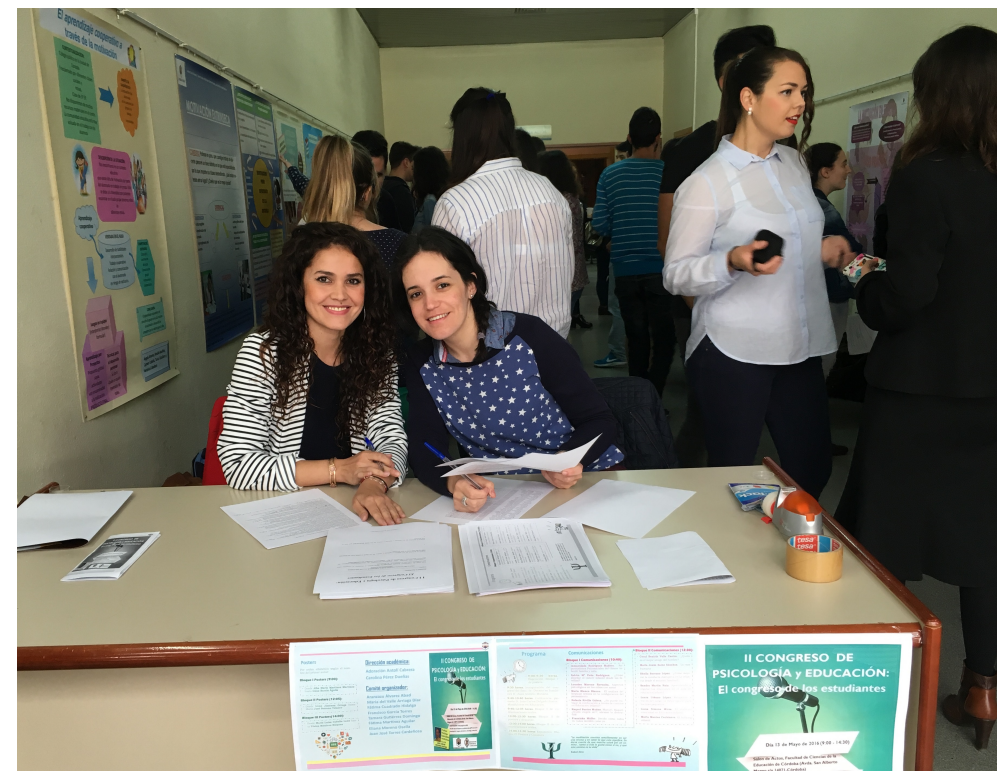

\begin{abstract}
3. Imagen de una sesión de póster del "II
Congreso de Psicología y Educación: El Congreso de los Estudiantes".
\end{abstract}

\title{
4. DESCRIPCIÓN
}

El congreso se desarrolla siguiento la estructura de un congreso científico habitual. Antes del mismo, se procede a la difusión de la actividad a través de distintos canales (moodle, cartelería, correo de difución de la Facultad, profeosrado, etc.) y a la inscripción de los participantes. El congreso se inicia con el reparto de documentación, que incluye el programa con las comunicaciones orales y sesiones de pósteres, y un plantilla para la valoración de las presentaciones por parte de los participantes. A continuación, se procede al acto de inauguración donde participan representantes de los órganos de gobierno de la Facultad y los organizadores. En los dos congresos desarrollados hasta el momento hemos contado con ponentes de reconocido prestigio que han profundizado en temas de interés para el alumnado en la conferencia inaugural. A partir de ese momento se suceden las comunicaciones orales y las sesiones de pósteres. Para concluir se recoge la información sobre la valoración de los asistentes de las comunicaciones y pósteres presentados. Con estos datos se procede a asignar los premios a los mejor valorados en las distintas categorías.

Todos los asistentes reciben su certificado de participación, y de autoría de presentaciones en ambos formatos, y los premiados reciben su certificado.

\section{RESULTADOS OBTENIDOS}

Con este proyecto hemos conseguido desarrollar una nueva forma de enseñanza dirigida a los Alumnos de Grado, siguiendo el informe Tunning que sugiere la necesidad de modificar el modelo tradicional de enseñanza con el fin de cambiar el énfasis exclusivo en la transmisión de contenidos para adoptar un modelo que incorpore de forma explícita la enseñanza de habilidades.

- Aprendizaje por descubrimiento para lograr el mayor acercamiento a un Currículo Transversal: enseñanza integradora de los conocimientos/habilidades de varias asignaturas.

- Enseñanzas motivadoras basadas en un sistema de tutorización, orientación y supervisión estrecha del trabajo del alumno, que se ve dirigido en la búsqueda de temáticas acerca de contenidos de las asignaturas y la confección de los temas.

- Estilos de enseñanza mediadora, lenguaje socrático, pensamiento crítico.

- Entrenamiento de la expresión oral. Práctica intensiva de habilidades de comunicación y lenguaje gestual, nogestual, dominio de la escena. Para dominar un tema hay que saber contarlo.

- Entrenamiento de la expresión escrita + escucha activa, ya que en cada sesión del congreso es preciso escribir al menos un párrafo sobre las presentaciones escuchadas. Críticas, dudas, o reflexiones valen para razonar sobre la temática y van al diario o portafolio de cada asignatura. 
- Aprendizaje experiencial y observacional. El/la estudiante aprende por propia exposición y por aprendizaje vicario, observando a sus compañeros.

- Entrenamiento y aprendizaje del uso creativo de la tecnología.

- Transmisión efectiva del discurso: 20\% Contar 80\% Demostrar.

- Incremento en Autoestima. Atribución propia a los resultados de sus propios logros.

- Potenciar la capacidad de aprender a aprender.

- Incremento de la sensación de automotivación.

- Facilitar la transferencia del conocimiento a nuevas situaciones.

- Hacer consciente al alumnado de que no hay soluciones fáciles a los problemas.

- Relación cercana alumno-profesor-profesional

- Inversión de roles (activo-pasivo).

- Lograr una meta final significativa para el alumno de todo un proceso de aprendizaje.

En definitiva, permitir una nueva concepción y mentalidad del alumnado, que se prepara para su ejercicio profesional desarrollando las habilidades, competencias y autoconfianza adecuadas.

\section{UTILIDAD}

La utilidad de la experiencia ha sido expresada tanto por el alumnado participante como por el profesorado. De forma resumida, se trata de contar con una herramienta para la docencia que permite de forma fluida la motivación y la implicación personal en alcanzar el propio conocimiento. Este tipo de enfoque del proceso enseñanza-aprendizaje facilita el abandono de roles pasivos y la asunción de un rol activo en el alumnado. En cuanto al profesorado, este tipo de metodología y práctica docente, le impulsa a salir de las situaciones de enseñanza tradicionales, y a focalizar el objetivo en la adquisición y puesta en práctica de competencias.

Por último, una de las ventajas del proyecto es que las prácticas adoptadas pueden incorporarse a cualquier otro contexto docente, en cualquier otra titulación o incluso en contextos no formales de aprendizaje. El único requisito es la implicación de los organizadores que deben tener un rol muy activo en este tipo de proyectos.

\section{CONCLUSIONES/DISCUSIÓN}

Con la puesta en práctica de este proyecto hemos obtenido un aumento de la implicación del alumnado, de la motivación y de la autonomía. Por otra parte, las competencias que eran objetivo se ven reforzadas con la práctica y pasan a ser un objetivo para el propio alumnado.

\section{AgRADECIMIENTOS}

A todos los participantes de los congresos, en cualquiera de los papeles y en cualquiera de la multitud de tareas que se han desarrollado. Gracias por vuestra generosidad. 


\section{BIBLIOGRAFÍA}

ALVERNO COLLEGE. Ability based learning program. Programa editado por el Alverno College, 2003.

BAIN, K. Lo que hacen los mejores profesores universitarios, Valencia, 2003.

BOYER COMMISSION ON EDUCATING UNDERGRADUATES IN THE RESEARCH UNIVERSIT. Reinventing undergraduate education: A blueprint for America's research Universities, Nueva York, 1998.

BRAUER, M. Enseñar en la Universidad. Consejos prácticos, destrezas y métodos pedagógicos. Madrid, 2013.

BRUNER, J.S. The process of education. Cambridge, 1960.

DE MIGUEL, M. (2009). "Métodos y modalidades de enseñanza en la educación superior.” Metodologías de enseñanza y aprendizaje para el desarrollo de competencias. Orientaciones para el profesorado universitario ante el espacio europeo de educación superior, Madrid, 2009, p.1726.

FINK, L. Creating Signicant Learning Experiences, San Francisco, 2003.

RUÉ, J. El Aprendizaje Autónomo en Educación Superior, Madrid, 2009

VALCÁRCEL, M. La preparación del profesorado universitario español para la convergencia europea en educación superior. Programa de estudios y análisis destinado a la mejora de la calidad de la enseñanza superior y de la actividad del profesorado universitario, Madrid, 2003. 